\title{
Zahnärzte haben den gesundheitsschädlichsten Job
}

Gesundheitliche Risiken sind von Beruf zu Beruf unterschiedlich: Dachdecker leben gefährlich, das ist klar. Sitzende Tätigkeiten gehen auf den Rücken, ebenso wie schweres Heben. Auch ständiger Wettereinfluss, Hitze, Kälte oder Strahlung bei der Arbeit machen sich gesundheitlich bemerkbar. Einer US-Analyse zufolge haben allerdings Zahnärzte den gesundheitsschädlichsten Job überhaupt, berichtet das Onlinemagazin Business Insider. In einem Ranking von 27 Berufen liegt der Zahnarzt unangefochten auf Rang eins der gesundheitlich risikoreichsten Berufe - deutlich vor anderen medizinischen Berufen oder auch Stahlarbeitern am Hochofen. Die Rangfolge wurde auf der Grundlage des Berufsinformationsnetzwerkes (Occupational Information Network) des US-Arbeitsministeriums erstellt und bezieht sich auf verschiedene, die Gesundheit beeinflussende Faktoren. Untersucht wurde unter anderem, wie stark Angehörige verschiedener Berufe Schadstoffen, Strahlung, (Infektions-) Krankheiten/Keimen oder anderen gefährlichen Gegebenheiten ausgesetzt sind. Aufgenommen in den Score wurde auch das Risiko, Verbrennungen, Schnitte oder Bisse zu erleiden, und wieviel Arbeitszeit im Sitzen verbracht wird. Vor allem hinsicht- lich Schadstoffen, Infektionskrankheiten und der Arbeit im Sitzen wird in der Analyse das Gesundheitsrisiko für Zahnärzte, aber auch für Zahntechniker und in den zahnärztlichen Assistenzberufen als sehr hoch bewertet.

sas

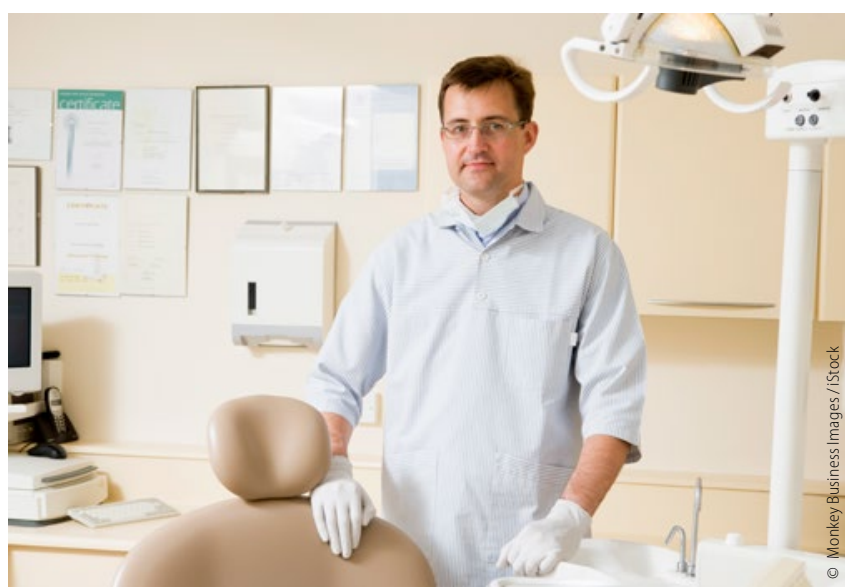

Neu auf dem YouTube-Kanal "FVDZ Presse“

\section{FVDZ Expertentipps sind jetzt online}

Unter dem Motto „Frag den FVDZ“ geben Referenten der FVDZ akademie ab sofort in einer neuen Reihe Expertentipps rund um die Zahnarztpraxis. In den etwa 15 Minuten langen Videoclips geht es um praktische Themen wie „Praxismietvertrag“ oder „Mein erster Job als Zahnarzt“. Die Videos sind für jeden kostenfrei zugänglich und werden in regelmäßigen $\mathrm{Ab}$ - ständen auf dem YouTube-Kanal „FVDZ Presse“ veröffentlicht. Im ersten Clip gibt Diana C. Brendel, Geschäftsführerin der FIBU-doc Praxismanagement GmbH und Referentin der FVDZ akademie, Tipps zum Thema „Praxisübernahme - was muss ich beachten?".

\section{Neues Dachmarkenkonzept des FVDZ}

\section{Kompetent, gebündelt, übersichtlich}

Der Freie Verband Deutscher Zahnärzte (FVDZ) bündelt seine Angebote für Mitglieder jetzt unter vier Dachmarken: FVDZ akademie, FVDZ ökonomie, FVDZ media und FVDZ praxis. Dieses Konzept findet sich ab sofort auch online im Internetauftritt des FVDZ wieder. Unter www.fvdz.de präsentiert sich der Freie Verband mit einer gestrafften und übersichtlichen $\mathrm{Na}$ vigation. Auf einen Blick können Interessierte die neuen Dach-
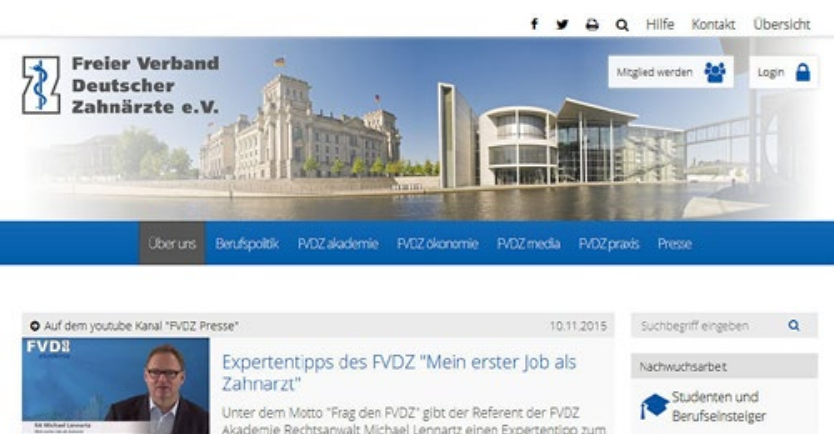

Expertentipps des FVDZ "Mein erster Job als Zahnarzt" Zahnarzt

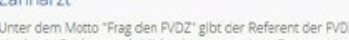

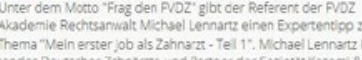

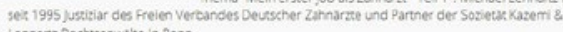
Lennarz Rectrsarwatte in Bonn.

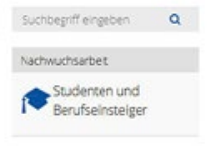

marken finden und mit nur einem Klick sehen, was sie bieten.

FVDZ akademie fasst alle Angebote zur Fortbildung zusammen, dazu gehören Seminare und Kongresse. Die Dachmarke FVDZ ökonomie versammelt wirtschaftliche Vorteilsangebote für Mitglieder, wie das Dynadent Bestellcenter, Versicherungen und das Studentenförderprogramm. Unter FVDZ media gibt es Publikationen vom Newsletter FVDZ aktuell bis hin zur Verbandszeitschrift Der Freie Zahnarzt. FVDZ praxis bietet Unterstützungsangebote und Hilfsmittel rund um die Praxisführung, -gründung und -abgabe. Dazu gehören das Nachschlagewerk Praxishandbuch, das Onlinecontrolling FVDZ control doc und die Hotlines zu Steuer, GOZ- und Rechtsfragen. Der neue moderierte Praxispool hilft bei der Praxisübergabe/Praxisübernahme.

Auf www.fvdz.de können sich User schnell und umfassend über alle Angebote des Freien Verbandes informieren. 